\title{
MiRNA-6089 inhibits rheumatoid arthritis fibroblast- like synoviocytes proliferation and induces apoptosis by targeting CCR4
}

\section{Suxian Lin}

The Third Clinical of Wenzhou Medical University, Wenzhou People's Hospital

\section{Zhiyong Zhang}

The Third Clinical College of Wenzhou Medical University, Wenzhou People's Hospital

\section{Shengnan Wang}

The Third Clinical College of Wenzhou Medical University, Wenzhou People's Hospital

\section{Yang Lu}

The Third Clinical College of Wenzhou Medical University, Wenzhou People's Hospital

\section{Meilv Yang}

The Third Clinical College of Wenzhou Medical University, Wenzhou People's Hospital

\section{Ping Chen}

The Third Clinical College of Wenzhou Medical University, Wenzhou People's Hospital

\section{Mudan Wang ( $\nabla$ peony_doc@126.com )}

The Third Clinical College of Wenzhou Medical University, Wenzhou People's Hospital https://orcid.org/0000-0001-5577-8707

\section{Research article}

Keywords: MiRNA-6089; Rheumatoid arthritis; Fibroblast-like synoviocytes; CCR4

Posted Date: April 10th, 2020

DOI: https://doi.org/10.21203/rs.3.rs-20663/v1

License: (a) (1) This work is licensed under a Creative Commons Attribution 4.0 International License. Read Full License

Version of Record: A version of this preprint was published at Archives of Physiology and Biochemistry on June 17th, 2020. See the published version at https://doi.org/10.1080/13813455.2020.1773862. 


\section{Abstract}

Background: Growing data have indicated that fibroblast-like synoviocytes (FLS) and miRNAs are implicated in the pathogenesis of rheumatoid arthritis (RA). This study was aimed to evaluate the function of miR-6089 in the regulation of RA-FLSs.

Methods: The expression of miR-6089 was measured by quantitative real time PCR (qRT-PCR). The RAFLSs were transfected with si-CCR4 plasmids or miR-6089 mimic, and subjected to CCK-8 and flow cytometry to analyze proliferation and apoptosis. The concentrations of MMP-1, TNF- $a$ and IL-6 in RAFLSs supernatant were detected using ELISA. The protein expression of caspase- $3,-8$ and -9 was detected using western blot.

Results: The levels of miR-6089 were detected to be significantly lower in the synovial tissues and FLSs of RA than in the synovial tissues and FLSs of healthy controls. The miR-6089 up-regulation in RA-FLSs significantly inhibited the proliferation and promoted cell apoptosis accompany with an increase protein expression of caspase-3, -8 and -9 . Furthermore, CCR4 was determined to directly target miR-6089, and its expression was significantly increased in the synovial tissues of RA than in the synovial tissues of healthy controls. Moreover, CCR4 overexpression effectively reversed the effect on proliferation and apoptosis induced by miR-6089 in RA-FLSs.

Conclusion: Our results revealed that miR-6089 may be a potential target for RA prevention and therapy of RA.

\section{Background}

Rheumatoid arthritis (RA) is a chronic, progressive, systemic, inflammatory joint disease characterized by synovial hyperplasia, inflammation, cartilage degradation, and bone destruction of unknown etiology[1]. The number of cases in developing countries reaches 5-50 per 100,000, while the incidence of adults in developed countries reaches $0.5 \%-1 \%[2]$. The incidence of RA is less than 15 years of age, but it has steadily increased with age[3]. Women are 3-5 times more likely than men[4]. It is well known that the abnormal activation of fibroblast-like synoviocytes (FLS) plays an important role in the initial stages of synovial inflammation and joint destruction as well as in the progressive development stage[5, 6]. Therefore, it is essential to identify novel biomarkers of RA progression in order to improve therapeutic strategies.

MicroRNAs (miRNAs) are endogenous small RNAs of about 20-24 nucleotides in length that can bind to the $3^{\prime}$ untranslated region (UTR) of a target mRNA through complete or incomplete complementary basepairing, and either degrade the transcripts or inhibit their translation[7]. Although miRNAs comprise only $2 \%$ of the human genome, they regulate the expression of nearly one-third of the genes, especially those involved in cell differentiation, cell proliferation and apoptosis and immune response[8]. Recently, a variety of abnormally expressed miRNAs have been found in RA peripheral blood mononuclear cells and 
FLS[9], but the specific mechanism involved in regulating this process and the mechanism by which abnormally expressed miRNAs are involved in regulating the occurrence of RA development is unclear.

Therefore, in this study, we detected the expression of miR-6089 in synovial tissues of healthy and RA and FLSs, and evaluated the effects of miR-6089 on proliferation, apoptosis and inflammatory response in RA-FLS. In addition, we also explored the role of miR-6089 in RA-FLSs, and the underlying molecular mechanisms were determined in order to provide a basis for future clinical applications for RA.

\section{Materials And Methods Samples of tissues}

The synovial tissues from 25 RA patients were obtained during knee replacement surgery. RA was diagnosed according to the American College of Rheumatology criteria for classification of disease. Healthy synovial tissues from 16 patients with a traumatic knee condition were collected as the normal control. The study was approved by the Ethics Committee of The Third Clinical College of Wenzhou Medical University, Wenzhou People's Hospital, and all subjects provided informed consent.

\section{Cell Culture And Transfection}

The normal human FLSs and RA-FLSs were obtained from Cell Applications (San Diego, CA, U.S.A.). The frozen stocks were routinely resuscitated and cultured in DMEM medium containing $10 \%$ fetal bovine serum (FBS) with $5 \% \mathrm{CO}_{2}$ in $37^{\circ} \mathrm{C}$. RA-FLSs cells were transfected with miR-6089 mimic, control mimic (miR-NC), lentiviral vectors CCR4 (siRNA1, siRNA2) and empty lentiviral vectors (Scramble) using Lipofectamine 3000 (Invitrogen, Carlsbad, CA) as per the manufacturer's instructions.

\section{Cell Proliferation}

Cell proliferation was detected by Cell Counting Kit-8 (CCK-8; Nanjing KeyGen Biotech, Co., Ltd., Nanjing, China). Concisely, cells were harvested and seeded in a 96-well plate $\left(2 \times 10^{3}\right.$ cells/well). Following overnight incubation, $10 \mu \mathrm{L}$ CCK-8 reagent was added to each well and the absorbance at $450 \mathrm{~nm}$ was determined after culturing for $24 \mathrm{~h}, 48 \mathrm{~h}$ and $72 \mathrm{~h}$ according to the manufacturer's protocol.

\section{Cell Apoptosis}

The cells were collected, washed twice with cold $1 \times$ PBS and resuspended in $100 \mu \mathrm{L}$ binding buffer, followed by incubation with Annexin V-FITC and propidium iodide for $15 \mathrm{~min}$ at room temperature in the dark. Next, $200 \mu \mathrm{L}$ of binding buffer was added, and the cells were analyzed by flow cytometry. 


\section{Luciferase Reporter Assay}

According to TargetScan (http://www.targetscan.org) and MiRanda

(http://www.microrna.org/microrna/home.do) databases, CCR4 was predicted as a putative target of miR-6089, which was verified using the dual luciferase reporter assay. Briefly, $2 \times 10^{4}$ cells $/ 96$ well in the logarithmic growth phase was cultured overnight, then 100 ng WT-CCR4-3'UTR, and MUT-CCR4-3'UTR luciferase reporter plasmid was co-transfected with $50 \mathrm{ng}$ miR-6089 mimic or NC using Lipofectamine 3000 , and the cells were cultured for $48 \mathrm{~h}$. The relative luciferase activity was detected according to the dual luciferase assay kit instructions. Five replicates were tested per condition.

\section{qRT-PCR analysis}

Total RNA was extracted from collected cultured FLSs and tissue samples utilizing a Trizol reagent (Invitrogen, Carlsbad, CA), and the purity and concentration of RNA were determined with a spectrophotometer. The RNA was reversely transcribed into CDNA templates used for real-time RT-PCR, which was then performed on an 7500 real-time PCR system (ABI, Foster City, CA) utilizing a SYBR Premix kit (TaKaRa, Tokyo, Japan) to measure the relative expression of, miR-6089, and CCR4 according to the $2^{-\triangle \Delta C t}$ method. The primer sequences were as follows: miR-6089-F-5'-CCC CTT CCG TGC GCC AGT GG-3', miR-6089-R-5'-AGG CCG GGG TGG GC-3'; CCR4-F-5'-CCC ACG GAT ATA GCA GAC ACC-3', CCR4-R-5'-GTG CAA GGC TTG GGG ATA CT-3'; U6-F-5'-GCT TCG GCA GCA CAT ATA CTA AAA T-3', U6-R-5'-CGC TTC ACG AAT TTG CGT GTC AT-3'; GAPDH-F-5'-AGA AGG CTG GGG CTC ATT TG-3', GAPDH-R-5' AGG GGC CAT CCA CAG TCT TC-3'. U6 or GAPDH were used as the internal reference for miR-625 or CCR4, and the relative expression levels were analyzed using the $2^{-\Delta \Delta C t}$ method.

\section{Elisa Assays}

The concentrations of MMP-1, TNF- $a$ and IL- 6 in the cell culture supernatant were detected using ELISA kits (Affymetrix, Santa Clara, CA, USA) following the manufacturer's recommendations.

\section{Western Blot Analysis}

To prepare protein extracts, harvested cells were washed thrice with chilled PBS, and lysed with RIPA buffer supplemented with protease inhibitors. The extracted protein was quantified using the BCA method, and the cell lysates were diluted in $5 \times$ loading buffer at the ratio of $1: 4$. After denaturing at $95^{\circ} \mathrm{C}$ for $10 \mathrm{~min}, 30 \mu \mathrm{g}$ protein per sample was subjected to SDS-PAGE. The protein bands were transferred to PVDF membranes and the latter were blocked with 5\% skim milk at room temperature for $2 \mathrm{~h}$. The blots were incubated overnight with primary antibodies against CCR4 (1:1000), Caspase-3(1:1000), Caspase- 
8(1:1000), Caspase-9 (1:1000), and GAPDH (1:5000) at $4{ }^{\circ} \mathrm{C}$ on a shaker. After washing thrice with TBST buffer, the blots were probed with HRP-conjugated secondary antibody (1:5000) for $1 \mathrm{~h}$ at room temperature, followed by 3 washes with TBST. ECL chromogenic substrate was used to visualize the bands, and the Image $J$ software was used to determine the gray value of each band, and the content of each protein was calculated relative to that of GAPDH.

\section{Statistical Analyses}

SPSS 19.0 was used for statistical analysis. Descriptive statistics were used to determine the group means and standard deviations in the numerical data. Independent sample t-test was used to compare two groups, and one-way ANOVA along with Dunnett's or Bonferroni's test was used for multiple groups. The level of significance was set to $p<0.05$.

\section{Results}

\section{miR-6089 was down-regulated in RA synovial tissues and FLSs}

The expression levels of miR-6089 were significantly lower in synovial tissues of RA patients versus the healthy controls $(P<0.01$; Fig. $1 \mathrm{~A})$. Consistent with the patient samples, the human RA-FLSs showed dramatically lower levels of miR-6089 compared to the normal human FLSs $(P<0.01$; Fig. 1B). Accordingly, it indicated that miR-6089 might process an important role in the pathogenesis of RA.

\section{Up-regulation of miR-6089 suppresses the proliferation and promotes the apoptosis in RA-FLSs}

To determine the effects of miR-6089 on RA-FLSs proliferation, and apoptosis, miR-6089 mimic was firstly used to transfect RA-FLSs with the miR-6089 mimic, which showed a 5.7-fold increase in miR-6089 expression $(P<0.01$; Fig. $2 A)$. The results from CCK-8 assay suggested that miR-6089 overexpression markedly suppressed the proliferation of RA-FLSs (Fig. 2B).

Next, flow cytometry was performed to detect the apoptosis after miR-6089 overexpression in RA-FLSs, indicating that miR-6089 overexpression significantly increased apoptosis ratio compared to the miR-NC group ( $\mathrm{P}<0.01$; Fig. $2 \mathrm{C}$ ). Mechanistically, we also found the increased protein expression of caspase-3, -8 and -9 in miR-6089 overexpressing RA-FLSs (Fig. 2D). Overall, our findings indicated that miR-6089 could inhibit the proliferation and trigger apoptosis of RA-FLSs. 


\section{Up-regulation of miR-6089 inhibits the release of inflammatory cytokines in RA-FLSs}

Next, we determined the role of miR-6089 in the response to the inflammatory cytokines of RA-FLSs. As shown in Fig. 3, miR-6089 overexpression significantly the release of the MMP-1, TNF-a and IL-6, suggesting that miR-6089 might protect the RA though inhibiting the inflammatory secretion.

\section{Mir-6089 Acts Directly On Ccr4 In Ra-flss}

The TargetScan database predicted that the 3'-UTR of the CCR4 gene was complementary to miR-6089 indicating that CCR4 is a putative target of miR-6089 (Fig. 4A). As shown in Fig. 4B, the possible relationship was validated by dual luciferase gene reporter assay which showed that the luciferase activity markedly reduced when co-transfected with the miR-6089 mimic and WT CCR4, whereas cotransfection with miR-6089 mimic and MUT CCR4 had no significant effect on the luciferase activity. Moreover, In contrast with the miR-NC group, there was a lower level ofCCR4 in miR-6089 overexpression group on both the mRNA and protein levels (Fig. 4C, D). Interestingly, CCR4 expression was significantly increased in RA synovial tissues (Fig. 4E). These results suggested that CCR4 is a target gene of miR6089 and is negatively regulated by the latter.

\section{Knocking down of CCR4 inhibits the proliferation and promotes the apoptosis in RA-FLSs}

First, the CCR4 expression in RA-FLSs was silenced by transfection of siRNA of CCR4. Two independent CCR4 siRNAs were used in case of off-target effect. Both siRNAs successfully knocked down CCR4 expression, with a scrambled sequence as control (Fig. 5A). The proliferation was detected with the CCK8 assay, which demonstrated that silencing CCR4 could significantly inhibit the cell proliferation (Fig. 5B). Flow cytometry was carried out 48 hours after the transfection of siRNAs. Both siRNAs of CCR4 increased the cell apoptosis (Fig. 5C), indicating the important role of CCR4 in RA-FLSs.

\section{CCR4 overexpression reverses the impact of miR-6089 on the proliferation and apoptosis of RA-FLSs}

The biological relevance of the miR-6089/CCR4 axis in RA-FLSs was further analyzed by co-transfecting with miR-6089 mimic and the CCR4 overexpression plasmid in RA-FLSs. As shown in Fig. 6A, the miR6089 mimic significantly decreased the levels of CCR4 ( $P<0.01)$. In addition, overexpression of CCR4 also abrogated the decreased proliferation and increased apoptosis induced by miR-6089 in RA-FLSs (Fig. 6B-C). Thus, miR-6089 might exert a suppressive role in RA-FLSs by repressing CCR4. 


\section{Discussion}

Accumulating evidence suggests that miRNAs regulate $1 / 3$ rd of the human genome via translational inhibition or degradation of the cognate genes, and form complex regulatory networks that have been implicated in malignant progression[10, 11]. Aberrant expression of miRNAs has been detected in RAassociated inflammatory innate immune responses, and they are increasingly being considered as prognostic biomarkers as well as therapeutic targets[12, 13]. For example, Yu et al. showed that miR-92a could target AKT2 to inhibit fibroblast-like synoviocyte proliferation and migration in RA[14]. Liu et al. found a decreased miR-125 level in synovial tissues, and miR-125 may regulate PI3K/Akt/mTOR pathway by directly inhibiting PARP2 expression, thereby weakening the development of RA[15]. Li et al also reported that miR-19 expression was significantly decreased in FLS from RA patients compared with healthy controls. MiR-19 inhibits the release of cytokine in FLS by targeting TLR2 in RA[16]. Dysregulation of miRNAs is established to be involved in the many diseases. miR-6089 serves a tumorsuppressive miRNA in ovarian cancer, which directly targets MYH9 to inactivate the Wnt/ $\beta$-catenin and its downstream EMT[17]. In our study, we proved that miR-6089 was significantly decreased in synovial tissues and FLS of RA patients compared with health donor. In vitro studies further showed that the recovery of miR-6089 significantly inhibited the proliferation accompany with an increased apoptosis in RA-FLSs. These results indicated that miR-6089 might play an important role in progression of RA. Further, the overexpression of miR-6089 notably increased the apoptosis with the apoptosis-related protein expression of caspase-3, -8 and -9 in RA-FLSs. Moreover, we found a lower level of inflammatory cytokines in RA-FLSs transfected with miR-6089 mimic compared with the negative control. Thus, miR6089 processes the inhibitive effect in the development of RA-FLSs.

Although the etiology and pathological mechanism of RA is still unknown, more and more reports confirm that miRNAs are closely related to the pathological process of RA[18, 19]. Different miRNAs can target and regulate different target genes to affect the pathological process of RA[20]. Thus, to further understand the underlying mechanisms by which miR-6089 inhibits the growth of RA-FLSs, we sought to identify its direct target genes. Bioinformatics analysis predicted CCR4 as a target of miR-62089, and the dual luciferase gene reporter assay further confirmed that CCR4 was negatively regulated by miR-6089. Meanwhile, in RA-FLSs, miR-6089 overexpression markedly decreased CCR4 expression. All of these results indicated the targeted binding correlation between miR-6089 and CCR4 in RA-FLSs.

Recently, studies showed that CCR4 is highly expressed on circulating Tregs[21]. Tregs is recruited at tumor sites in many cancers, such as breast cancer[22] and colorectal cancer[23]. The anti-CCR4 antibody, alone or in combination with other immune modulators, may potentially be used in the treatment of a human solid cancer with high levels of CCR4 expression in tumor-infiltrating leukocytes and abnormal plasma CCR4 ligand levels[24]. Importantly, CCR4 has been widely implicated in the pathogenesis of inflammatory diseases such as asthma and atopic dermatitis due to its expression on Th2 cells[25].Interestingly, it was found an increased mRNA level of CCR4 in synovial tissues. Notably, overexpression of CCR4 reversed the effects of miR-6089 on proliferation and apoptosis in RA-FLSs by. 
Our results showed that miR-6089 performed its inhibitive role in RA-FLSs, at least in part, by targeting CCR4.

In conclusion, miR-6089 is found to be frequently downregulated in RA synovial tissues and FLSs. And the overexpression of miR-6089 suppresses proliferation, promotes apoptosis associated with the apoptosis protein expression (caspase-3 -8 and -9 ), as well as decreases the secretion of inflammatory cytokines in RA-FLSs by targeting CCR4. Our findings provide new insights into the roles of miR-6089 and CCR4 in RA, and identify potential prognostic biomarkers and therapeutic targets.

\section{Abbreviations}

FLS: Fibroblast-like synoviocytes; RA: Rheumatoid arthritis; qRT-PCR: Quantitative real time PCR; miRNAs: MicroRNAs; UTR: Untranslated region; FBS: Fetal bovine serum; CCK-8: Cell Counting Kit-8

\section{Declarations}

\section{Acknowledgements}

Not applicable.

\section{Author contributions}

S.L. wrote the manuscript and operated the experiments; Z.Z., S.W., Y.L. and M.Y. operated molecular experiments; P.C. and M.W. analysed the data; S.L. and M.W. designed the experiments and edited the manuscript.

\section{Funding}

This study was supported by Wenzhou Science and Technology Bureau Project (Y20160301 and 2017Y0539).

\section{Availability of data and materials}

The datasets used and/or analyzed during the current study are available from the corresponding author on reasonable request.

\section{Ethics approval and consent to participate}

This study was approved by the ethics committee of the The Third Clinical College of Wenzhou Medical University, Wenzhou People's Hospital, and informed consent was signed by the volunteer and his family members.

\section{Consent for publication}


Not applicable.

Competing interests

The authors declare that they have no competing interests.

\section{References}

1. Yue T, Fan X, Zhang Z, Liu Z, Guo M, Bai F, Gong X, Gao C, Xiao L: Downregulation of IncRNA ITSN1-2 correlates with decreased disease risk and activity of rheumatoid arthritis (RA), and reduces RA fibroblast-like synoviocytes proliferation and inflammation via inhibiting NOD2/RIP2 signaling pathway. Am J Trans/ Res 2019, 11(8):4650-4666.

2. Zhang L, Zhu M, Li M, Du Y, Duan S, Huang Y, Lu Y, Zhang J, Wang T, Fu F: Ginsenoside Rg1 attenuates adjuvant-induced arthritis in rats via modulation of PPAR-gamma/NF-kappaB signal pathway. Oncotarget 2017, 8(33):55384-55393.

3. Wroblewski BM, Siney PD, Fleming PA: Charnley low-frictional torque arthroplasty in young rheumatoid and juvenile rheumatoid arthritis: 292 hips followed for an average of 15 years. Acta orthopaedica 2007, 78(2):206-210.

4. Humphreys J, Verheul M, Barton A, Fu B, Toes R, Symmons D, Trouw L, Verstappen S: Association of anti-carbamylated protein antibodies with long-term disability and increased disease activity in patients with early inflammatory arthritis: results from the Norfolk Arthritis Register. Lancet 2015, 385 Suppl 1:S44.

5. Li S, Chen JW, Xie X, Tian J, Deng C, Wang J, Gan HN, Li F: Autophagy inhibitor regulates apoptosis and proliferation of synovial fibroblasts through the inhibition of PI3K/AKT pathway in collageninduced arthritis rat model. Am J Trans/ Res 2017, 9(5):2065-2076.

6. Wang Y, Feng T, Duan S, Shi Y, Li S, Zhang X, Zhang L: miR-155 promotes fibroblast-like synoviocyte proliferation and inflammatory cytokine secretion in rheumatoid arthritis by targeting FOXO3a. Experimental and therapeutic medicine 2020, 19(2):1288-1296.

7. Li Y, An H, Pang J, Huang L, Li J, Liu L: MicroRNA profiling identifies miR-129-5p as a regulator of EMT in tubular epithelial cells. Int J Clin Exp Med 2015, 8(11):20610-20616.

8. Li H, Yu L, Li M, Chen X, Tian Q, Jiang Y, Li N: MicroRNA-150 serves as a diagnostic biomarker and is involved in the inflammatory pathogenesis of Parkinson's disease. 2020:e1189.

9. Zhao W, Li D, Su Y, Zhao H, Pang W, Sun Y, Wu S: MicroRNA-147 negatively regulates expression of toll-like receptor-7 in rat macrophages and attenuates pristane induced rheumatoid arthritis in rats. Am J Transl Res 2019, 11(4):2219-2231.

10. Chen B, Xia Z, Deng YN, Yang Y, Zhang P, Zhu H, Xu N, Liang S: Emerging microRNA biomarkers for colorectal cancer diagnosis and prognosis. Open biology 2019, 9(1):180212.

11. Hunter S, Nault B, Ugwuagbo KC, Maiti S, Majumder M: Mir526b and Mir655 Promote Tumour Associated Angiogenesis and Lymphangiogenesis in Breast Cancer. Cancers 2019, 11(7). 
12. Liu H, Zhu Y, Gao Y, Qi D, Zhao L, Zhao L, Liu C, Tao T, Zhou C, Sun X et al: NR1D1 modulates synovial inflammation and bone destruction in rheumatoid arthritis. Cell Death Dis 2020, 11(2):129.

13. Simon D, Kleyer A: Micro-structural bone changes are associated with broad-spectrum autoimmunity and predict the onset of meumatoid arthritis. 2020.

14. Yu FY, Xie CQ, Jiang CL, Sun JT, Feng HC, Li C, Huang XW: MiR-92a inhibits fibroblast-like synoviocyte proliferation and migration in rheumatoid arthritis by targeting AKT2. Journal of biosciences 2018, 43(5):911-919.

15. Liu K, Zhang Y, Liu L, Yuan Q: miR-125 regulates PI3K/Akt/mTOR signaling pathway in rheumatoid arthritis rats via PARP2. Biosci Rep 2019, 39(1).

16. Li Z, Cai J, Cao X: MiR-19 suppresses fibroblast-like synoviocytes cytokine release by targeting toll like receptor 2 in rheumatoid arthritis. Am J Trans/ Res 2016, 8(12):5512-5518.

17. Liu L, Ning Y, Yi J, Yuan J, Fang W, Lin Z, Zeng Z: miR-6089/MYH9/beta-catenin/c-Jun negative feedback loop inhibits ovarian cancer carcinogenesis and progression. Biomed Pharmacother 2020, 125:109865.

18. Arias de la Rosa I, Perez-Sanchez C, Ruiz-Limon P, Patino-Trives A, Torres-Granados C, JimenezGomez Y, Abalos-Aguilera MDC, Cecchi I, Ortega R, Caracuel MA et al: Impaired microRNA processing in neutrophils from rheumatoid arthritis patients confers their pathogenic profile. Modulation by biological therapies. Haematologica 2020.

19. Yang $P$, Zhang $M$, Wang $X$, Xu AL, Shen M, Jiang B, Zhou X, Zhou L: MicroRNA let-7g-5p alleviates murine collagen-induced arthritis by inhibiting Th17 cell differentiation. Biochem Pharmaco/2020, 174:113822.

20. Iwamoto N, Kawakami A: Recent findings regarding the effects of microRNAs on fibroblast-like synovial cells in rheumatoid arthritis. Immunological medicine 2019, 42(4):156-161.

21. Watanabe M, Kanao K: Increased infiltration of CCR4-positive regulatory T cells in prostate cancer tissue is associated with a poor prognosis. 2019, 79(14):1658-1665.

22. Xydia M, Hu X, Pincha M, Umansky L, Breyer C, Hillier M, Bonertz A.

23. Sasidharan Nair V, S MT, R ZT, Ahmed AA, Kurer MA, Murshed K, Soofi ME, Ouararhni K, N MA, Abu Nada M et al: Transcriptomic Profiling of Tumor-Infiltrating CD4(+)TIM-3(+) T Cells Reveals Their Suppressive, Exhausted, and Metastatic Characteristics in Colorectal Cancer Patients. Vaccines 2020, 8(1).

24. Berlato C, Khan MN, Schioppa T, Thompson R, Maniati E, Montfort A, Jangani M, Canosa M, Kulbe H, Hagemann UB et al: A CCR4 antagonist reverses the tumor-promoting microenvironment of renal cancer. The Journal of clinical investigation 2017, 127(3):801-813.

25. Anderson CA, Patel P, Viney JM, Phillips RM, Solari R, Pease JE: A degradatory fate for CCR4 suggests a primary role in Th2 inflammation. 2020.

\section{Figures}


A

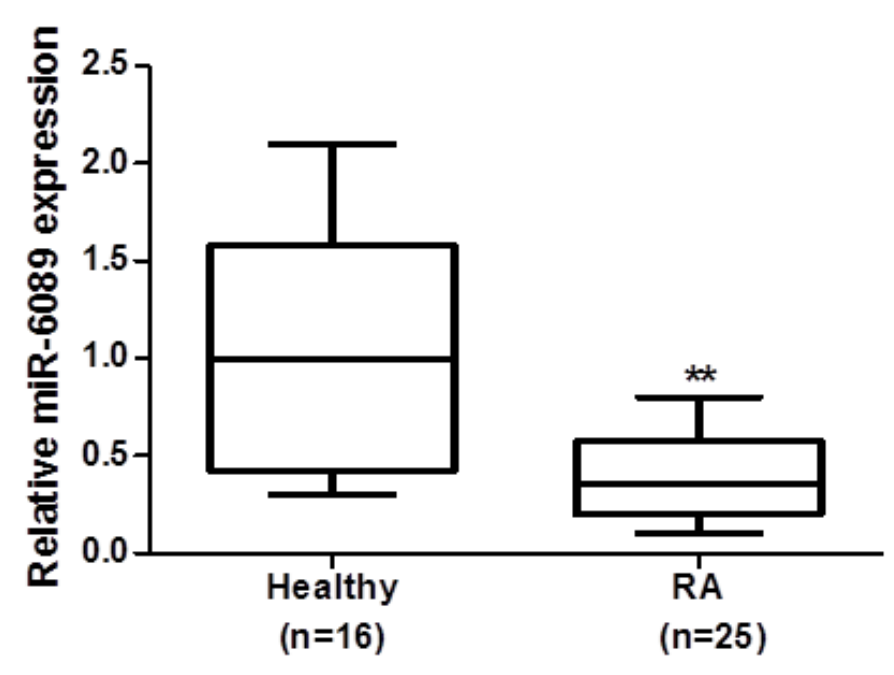

B

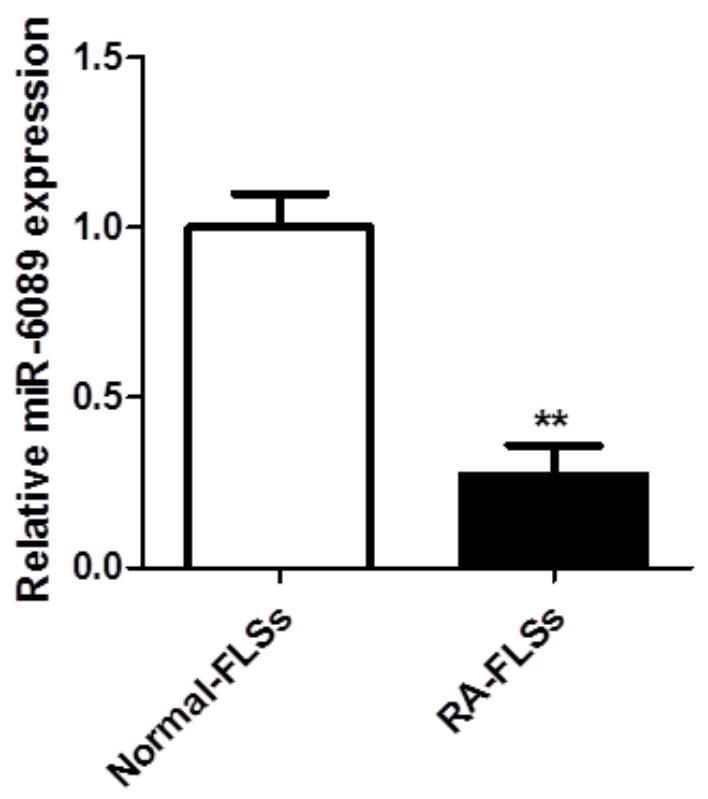

\section{Figure 1}

miR-6089 expression level is down-regulated in RA synovial tissues and FLSs (A) The relative expression of miR-6089 in synovial tissue from RA patients $(n=25)$ or healthy control (normal, $n=16)$ was examined by qRT-PCR. (B) The relative expression of miR-6089 in FLSs from RA patients (RA-FLSs) or healthy control (normal-FLSs) was examined by qRT-PCR. ${ }^{\star *} \mathrm{P}<0.01$ vs. Healthy-synovial or Normal-FLSs groups. 
A

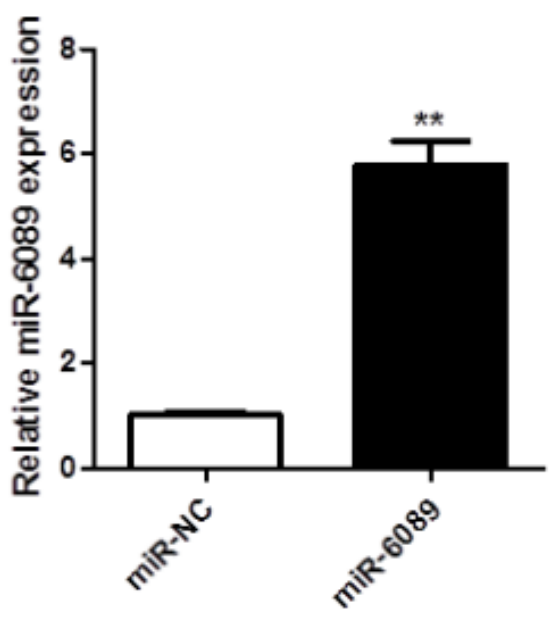

B

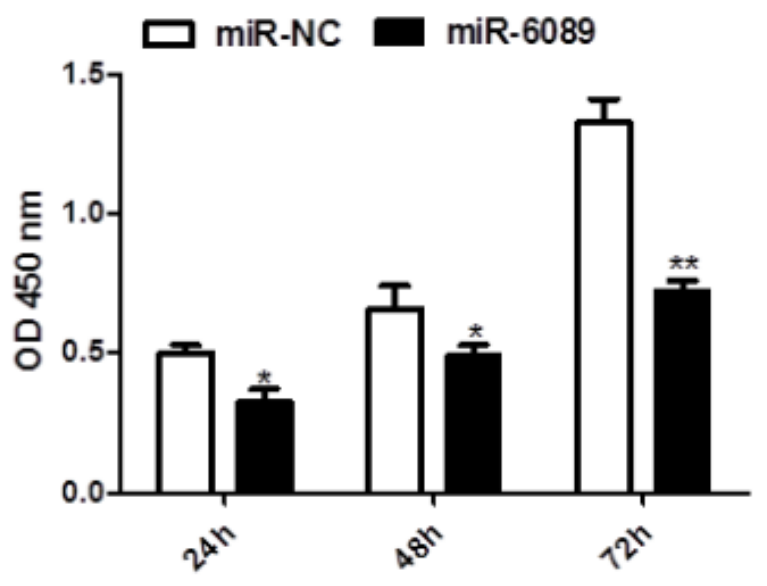

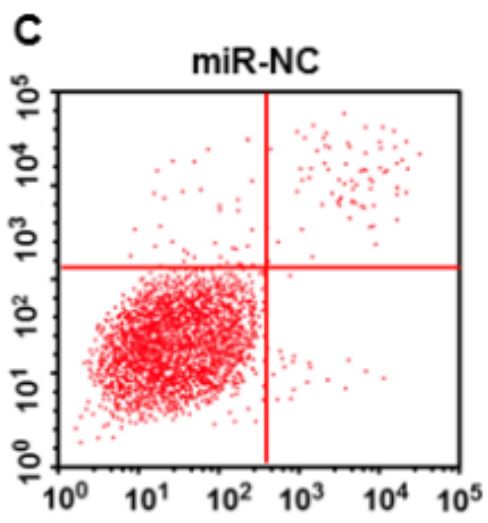
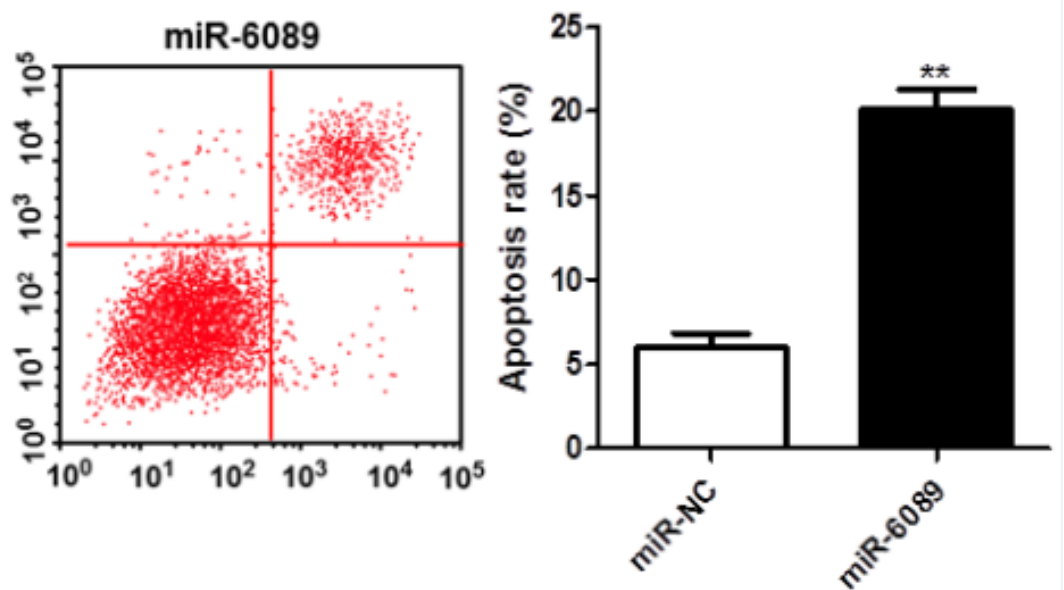

D
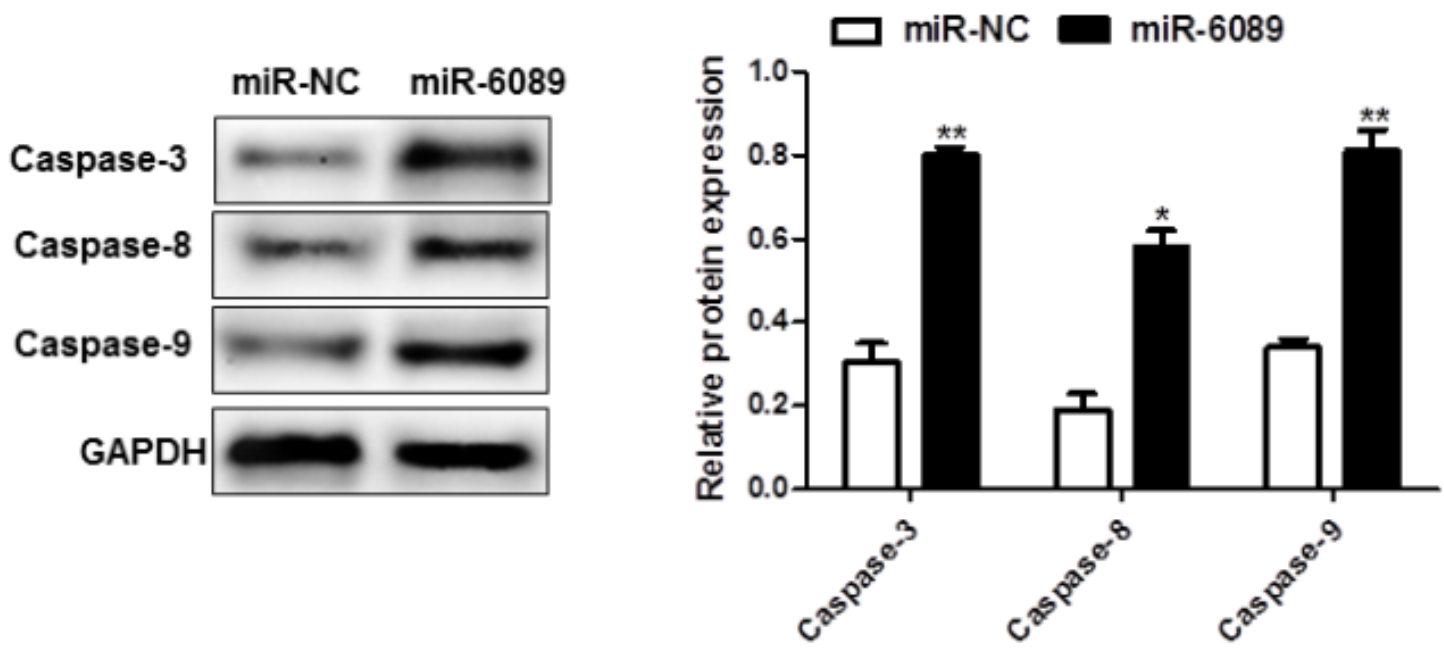

Figure 2

Up-regulation of miR-6089 suppresses the proliferation, promotes the apoptosis in RA-FLSs (A) miR-6089 expression levels were determined in RA-FLSs transfected with miR-6089 mimic or miR-NC mimic by qRTPCR. $(B, C)$ Cell proliferation and apoptosis were determined in RA-FLSs transfected with miR-6089 mimic or miR-NC. (D) The Caspase-3, -8 and -9 proteins expression were determined in RA-FLSs transfected with 
miR-6089 mimic or miR-NC by western blot. GAPDH was used to as internal control. *P<0.05; **P<0.01 vs. miR-NC group.
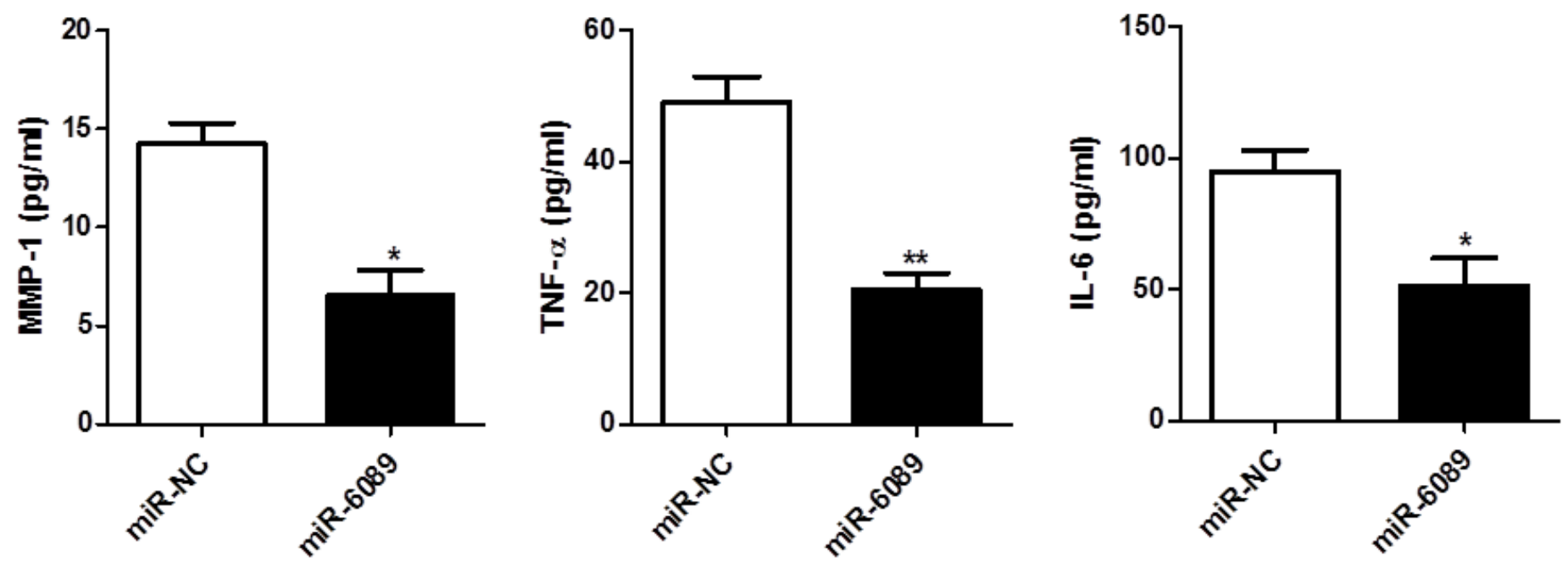

Figure 3

Up-regulation of miR-6089 suppresses inflammatory cytokines release of RA-FLSs. FLSs transfected with either miR-6089 or miR-NC were cultured for $24 \mathrm{~h}$. RA-FLS transfected with miR-506 mimic significantly reduced production of MMP-1, TNF- $a$, and IL-6 compared with RA-FLSs transfected with miR-NC. ${ }^{*}<0.05$, $\star * P<0.01$ vs. miR-NC group. 


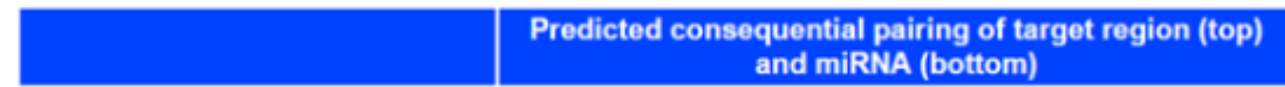

Position 1355-1361 of CCR4 3' UTR 5'

and miRNA (bottom)

B
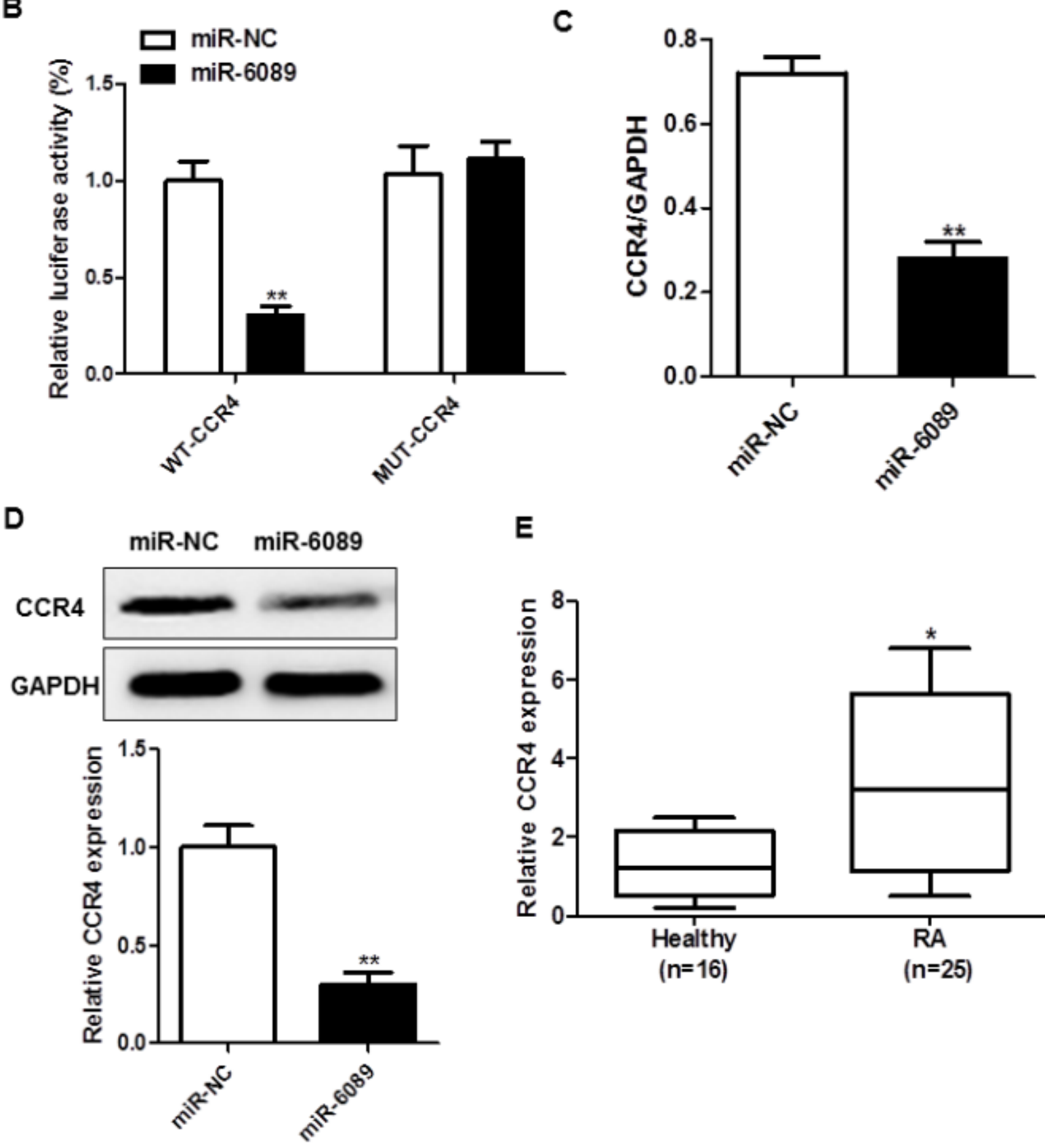

E

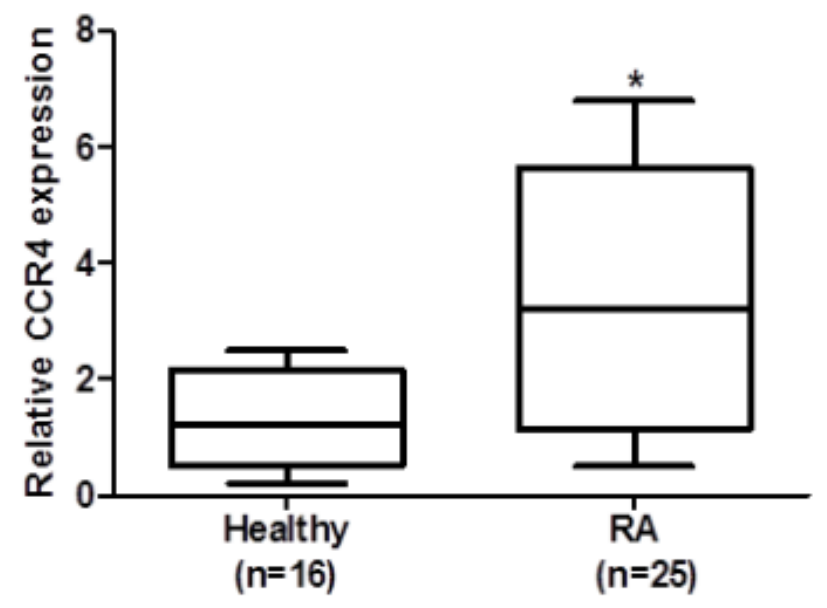

Figure 4

CCR4 is a direct target of miR-6089 in RA-FLSs. (A) Putative miR-6089-binding sites in the 3'-UTR of CCR4 were shown. (B) Relative luciferase activity was detected in RA-FLSs co-transfected with WT or MUT 3'UTR CCR4 reporter plasmids and miR-6089 mimic or miR-NC. (C, D) CCR4 expression on mRNA and protein levels was examined in RA-FLS. RA-FLSs transfected with miR-6089 mimic or miR-NC mimic. GAPDH was used as an internal control. (E) Relative CCR4 mRNA expression was detected in synovial 
tissues from RA patients and health donor. GAPDH was used as an internal control. ${ }^{\star} P<0.05,{ }^{\star *} P<0.01$ vs. miR-NC group or Healthy.
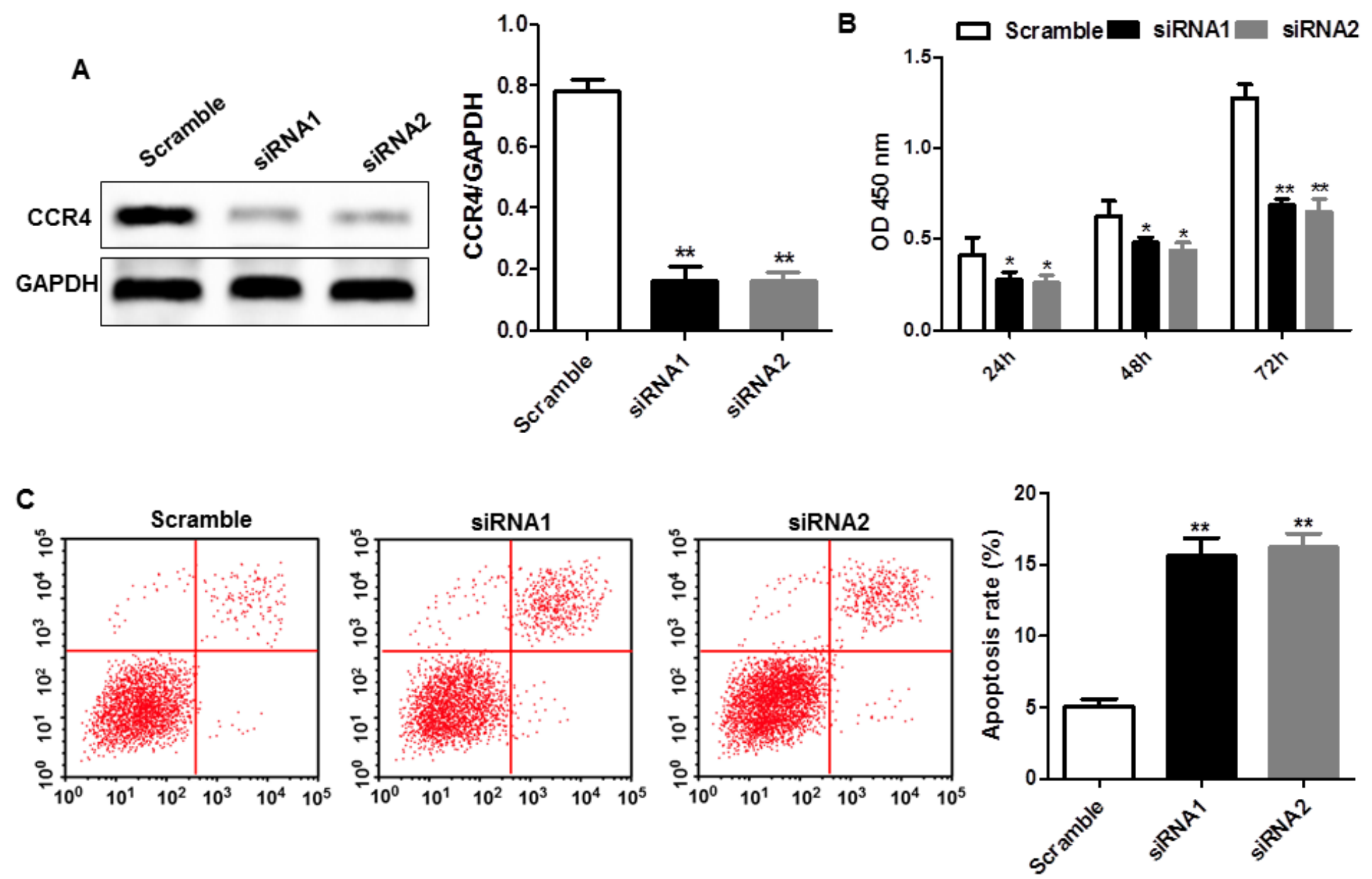

Figure 5

Silencing of CCR4 inhibits cell proliferation of RA-FLSs. RA-FLSs were transfected with control (scramble) or CCR4-targeting siRNAs (CCR4 siRNA1 and 2) for $48 \mathrm{~h}$. (A) Western blot analysis of CCR4 protein levels in RA-FLSs. (B) CCK8 assay was performed to detect cell proliferation after culturing for 24,48 or $72 \mathrm{~h}$. (C) Cell apoptosis was determined by flow cytometry. ${ }^{*} P<0.05,{ }^{*} P<0.01$ vs. miR-NC group. 

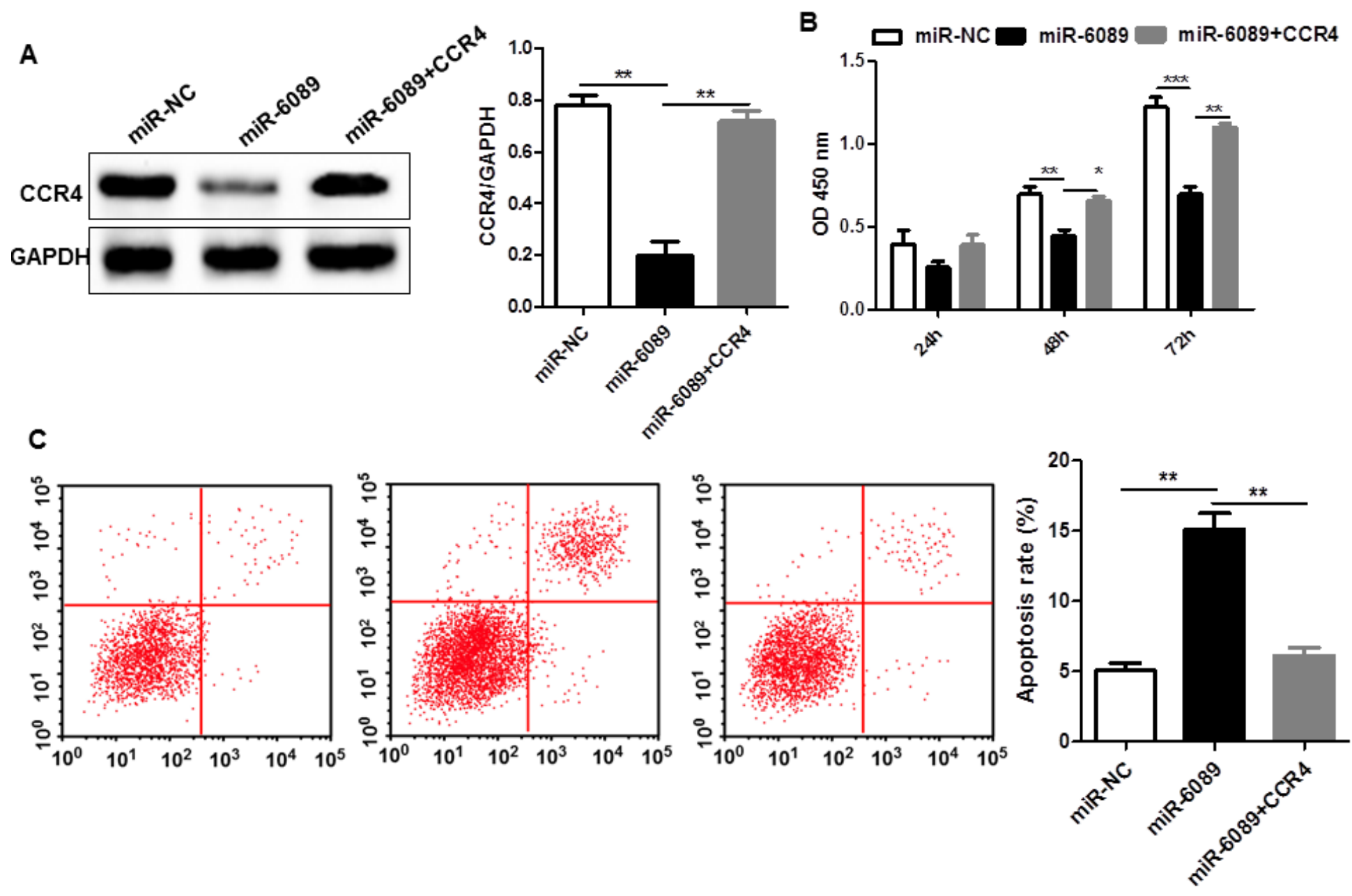

Figure 6

The impact of miR-6089 on the proliferation and apoptosis of RA-FLSs was reversed by CCR4 overexpression. (A) CCR4 protein expression was determined in RA-FLSs transfected with miR-6089 mimic or miR-NC and with/without CCR4 overexpression vector (lack of 3'-UTR). GAPDH was used as an internal control. (B, C) The effect of miR-6089 on cell proliferation and apoptosis in RA-FLSs was reversed under the condition of overexpression of CCR4. 